\title{
Correction: weight management interventions in adults with intellectual disabilities and obesity: a systematic review of the evidence
}

Dimitrios Spanos ${ }^{1,2}$, Craig Andrew Melville ${ }^{2}$ and Catherine Ruth Hankey ${ }^{3 *}$

\section{Correction}

Following the publication of this article [1], we noted errors to Table six (Table 1 here). Corrected version is presented below.

\footnotetext{
* Correspondence: Catherine.Hankey@glasgow.ac.uk

${ }^{3}$ Human Nutrition, College of Medical, Veterinary and Medical and Life

Sciences, University of Glasgow, Glasgow Royal Infirmary, Level 2, New Lister

Building, Glasgow G31 2ER, UK

Full list of author information is available at the end of the article
} 
Table 1 Multi-component interventions

\section{Study/ Location/ Type Participants}

Jackson 1982 [31] Gender: all females

Australia

\section{(a) Treatment group}

Community

Quasi-experimental study with a control group

Harris 1984 [32]

USA

Community

Quasi-experimental study with a comparison group $\mathrm{n}=6$

Weight status: $10 \%$ overweight Age (years), mean: 21.8

ID, mean IQ : 38.17

\section{(b) Control group}

$n=6$

Age (years), mean: 23.5

ID, mean IQ :40.33

Attrition/drop out: none

Total $n=21$

Weight status: not reported

\section{(a) Completers}

$\mathrm{n}=10$

Gender: 8 females,

2 males

Age (years) ${ }^{a}: 22.7(6.37)$

ID, IQ ${ }^{a}: 52.5$ (12.80)

(b) Non completers: 11

Attrition/drop out: 11

Intervention

Follow up

Results

Duration: 14 weeks of every 2 weeks

17 weeks

(a) Me

group sessions
by a teacher.

(a) 7 sessions with the parents, 6 sessions with group members and the teacher.

Diet: Advice on healthy eating diet, avoid

fad diets. Activity: General advice on

physical activity e.g. using stairs instead of

elevator. Behaviour: self-monitoring, reward,

punishment, change of rate of eating,

\section{reinforcement.}

\section{Maintenance: none reported}

(b) No intervention

(b) Mean weight change, kg: 0.00

Duration: 7 weekly group sessions and 1 hour booster session 26 weeks after the first session.

(a) Diet: education on healthy balanced diet, distinguishing high and low calorie foods, diabetic exchange diet (ADA, 1977). Activity: $5-10 \mathrm{~min}$ aerobic exercise at the end of session. Behaviour: stimulus control, self-monitoring, self-reinforcement, goal setting, self-contacting. Carers

attended the sessions.

Maintenance: none reported a) Mean weight change, kg: -7.33

Significant weight reduction of (a) across all the follow up

(a) Mean weight change, $\mathrm{kg}:-6.25$

(b) Mean weight change, $\mathrm{kg}:-0.59$

Mean weight change, kg: -6.08

(b) Mean weight change, $\mathrm{kg}:+0.33$

(b) Mean weight change, $\mathrm{kg}:+2.39$ $(p<0.05)$

$(p<0.05)$ (a) Mean weight change, kg:-3.0 $(p<0.05)^{b}$

(a) Mean weight change, $\mathrm{kg}:-0.76$
12 months 
Table 1 Multi-component interventions (Continued)

(a) participants with ID

Total $n=154$, final $n=92$

\section{Community}

Quasi-experimental study with a comparison group

\section{Study/Location/Type}

Mann 2006 [34]

USA

Community

Uncontrolled quasiexperimental study

Bazzano 2009 [35]
Weight status, BMI': 35.4 (7.0) Gender: $54.4 \%$ females Age (years) ${ }^{a}: 39.7(11.5)$ ID, IQ $: 50.2(14.3)$

Attrition/drop out: $18.8 \%$

(b) no ID

Total $n=270$, final $n=97$

Weight status, BMI :38.4 (8.6) Gender: $84.5 \%$ females Age (years) ${ }^{\mathrm{a}}: 49.9$ (11.48)

Attrition/drop out: $30 \%$

\section{Participants}

Total $n=324$, available data for 192

Weight status, BMI : $35.38(6.85)$

Gender:66.7 \% females, $33.3 \%$ males

Age (years) ${ }^{\mathrm{a}}: 38.6(11.5)$

ID, IQ $: 50.7$ (13.3)

Total $\mathrm{n}=85$ signed up, 44 completers
Attrition/drop out: $20 \%$
Duration: 8 week intervention. The "HELP"

tervention (Health Education Learning

Program) led by health educators. 8 group

sessions and 2 to 4 home visits.

Diet: a home visit to develop dietary plan and do a grocery visit. Activity: a home visit to develop an exercise programme e.g.

walking routes, optional brisk walk after the sessions. Behaviour: motivation to change, relapse prevention, avoidance of "automatic thinking".

Maintenance: none reported

2 months

(a) Mean BMl change, $\mathrm{kg} / \mathrm{m}^{2}: 0^{\mathrm{b}}$

No significant difference between (a) and (b)

(b) Mean BMl change, $\mathrm{kg} / \mathrm{m}^{2}:-0.89$

\section{Intervention}

Duration: 8 weekly group sessions $(90 \mathrm{~min}$ each) using health education "Steps to Your Health" led by trained staff. Included

home visits.

\section{Results}

Mean BMI change, $\mathrm{kg} / \mathrm{m}^{2}:-0.31^{\mathrm{b}}$ $(p<0.05)$

Diet: individual dietary plan and a grocery store visit. Activity: optional brisk walking and individual exercise programme.

Behaviour: motivation to change, relapse prevention, barriers to change.

Maintenance: none reported 
Table 1 Multi-component interventions (Continued)

\begin{tabular}{|c|c|c|c|c|}
\hline & & $\begin{array}{l}\text { Programme" focusing on health education } \\
\text { and peer mentoring. Phone calls included. }\end{array}$ & & \\
\hline USA & $\begin{array}{l}\text { Weight status: } 36.4 \% \text { overweight, } \\
38.6 \% \text { obese, } 18.2 \% \text { very obese }\end{array}$ & $\begin{array}{l}\text { Diet: education and cooking demonstration. } \\
\text { Activity: education and supervised physical } \\
\text { activity ( } 90 \mathrm{~min}) \text {. Exercise in local parks and } \\
\text { fitness facilities. Behaviour: behaviour } \\
\text { modification and reward systems. Carers } \\
\text { were encouraged to attend the sessions. }\end{array}$ & & $\begin{array}{l}\text { Mean BMl change, } \mathrm{kg} / \mathrm{m}^{2}:-0.5 \\
(p<0.05)^{\mathrm{b}}\end{array}$ \\
\hline Community & Gender: $61 \%$ females, $39 \%$ males & \multirow[t]{4}{*}{ Maintenance: none reported } & & \\
\hline \multirow{3}{*}{$\begin{array}{l}\text { Uncontrolled quasi- } \\
\text { experimental study }\end{array}$} & Age (years): 18-59 & & & \\
\hline & $\begin{array}{l}\text { ID: } 68 \% \text { mental retardation, } \\
25 \% \approx \text { mental retardation, Cerebral } \\
\text { palsy, epilepsy, and autism } \\
\text { diagnosed in } 15 \% \text { to } 20 \%\end{array}$ & & & \\
\hline & Attrition/ drop out: $35 \%$ & & & \\
\hline \multirow[t]{4}{*}{ Geller 2009 [36] } & \multirow[t]{4}{*}{ Total $n=45$} & \multirow{4}{*}{$\begin{array}{l}\text { (a): Empowerment model. 1st year: Twice } \\
\text { weekly sessions led by a physician ( } 60 \text { min } \\
\text { each): Group and individual sessions based } \\
\text { on the "Funk" model. Diet: meal planning, } \\
\text { cooking demonstrations. Activities: music } \\
\text { chairs, dancing, exercise to music. } \\
\text { Behaviour: Activities creating feelings of } \\
\text { community, feelings of success and of } \\
\text { being important. }\end{array}$} & & $\begin{array}{l}\text { (a) }+(\mathbf{b})(n=43) \text { Mean weight change, } \\
\text { kg: }-0.26^{b}\end{array}$ \\
\hline & & & 6 months & $\begin{array}{l}\text { (a) }+(\mathbf{b})(n=38) \text { Mean weight change } \\
\text { kg:-0.78 }\end{array}$ \\
\hline & & & 12 months & $\begin{array}{l}\text { (a) }+(\mathbf{b})(n=36) \text { Mean weight change, } \\
\text { kg:-0.74 }\end{array}$ \\
\hline & & & 18 months & $\begin{array}{l}(\mathbf{a})+(\mathbf{b})(n=14) \text { Mean weight change, } \\
\text { kg:-2.73 }\end{array}$ \\
\hline USA & Gender: 25 females, 18 males & $\begin{array}{l}\text { (b): 2nd year, once weekly group sessions } \\
\text {-same as (a) }\end{array}$ & & \\
\hline Community & $\begin{array}{l}\text { Weight status: obese/ } \\
\text { overweight:-Age (years): } \\
\text { average } 42.6\end{array}$ & Weight maintenance: none reported & & \\
\hline \multirow{2}{*}{$\begin{array}{l}\text { Uncontrolled quasi- } \\
\text { experimental study }\end{array}$} & ID: not reported & & & \\
\hline & $\begin{array}{l}\text { Attrition/ drop out: } 2 \text { dropped } \\
\text { out, } 14 \text { completed } 18 \text { month } \\
\text { measurements }\end{array}$ & & & \\
\hline Melville 2011 [37] & Total $n=54$ & $\begin{array}{l}\text { Duration: } 6 \text { months of } 9 \text { sessions every } \\
2-3 \text { weeks individual consultations } \\
(45-60 \text { min each) based on the GCWMS } \\
\text { led by a dietician and a medical graduate. }\end{array}$ & 6 months & $\begin{array}{l}\text { Mean weight change, } \mathrm{kg}(\mathrm{SD}):-4.47 \\
(4.45)(p<0.0001)^{\mathrm{b}}\end{array}$ \\
\hline UK & Weight status, BMI $: 40$ (8.03) & $\begin{array}{l}\text { Diet: } 600 \mathrm{kcal} / \mathrm{d} \text { energy deficit diet. } \\
\text { Activity: aim for } 30 \text { min of moderate } \\
\text { physical activity for } 5 \text { days per week. } \\
\text { Behaviour: goal setting, problem solving, } \\
\text { cue avoidance, stimulus control. Carers } \\
\text { were encouraged to assist if needed. }\end{array}$ & & $\begin{array}{l}\text { Mean BMl change, } \mathrm{Kg} / \mathrm{m}^{2}:-1.82 \\
(p<0.0001)\end{array}$ \\
\hline Community & $\begin{array}{l}\text { Gender:40.7 \% males, } 59.3 \% \\
\text { females }\end{array}$ & Maintenance: none reported & & \\
\hline
\end{tabular}

Maintenance: none reported 
Table 1 Multi-component interventions (Continued)

\begin{tabular}{ll}
\hline Uncontrolled quasi- & Age (years) ${ }^{\mathrm{a}}: 48.3(12.01)$ \\
experimental study & ID: $31.5 \%$ mild, $31.5 \%$ moderate, \\
& $35.2 \%$ severe, $1.9 \%$ profound. \\
& Attrition/drop out: 3 dropped out, \\
4 non completers on time
\end{tabular}

\section{Study/ Location/ Type}

Saunders 2011[38]

USA

Community
$\begin{aligned} & \text { Uncontrolled quasi- } \\ & \text { experimental study }\end{aligned}$
ID: not reported
Attrition/drop out: 73 six month
completers, 43 twelve month
completers

\section{Intervention}

Duration: 6 months of one individual session (60-90 $\mathrm{min})$ and monthly

consultations (30 min each) led by dietician behaviour analysts, physiologists.

Diet: a 1200 to $1300 \mathrm{kcal} / \mathrm{d}$ diet based on volumetrics, at least 5 portions of fruits and vegetables; up to three low-calorie, meal/snack-replacement shakes; two packaged entrees of less than 300 calories each and other low calorie items, 2 shake mixes from Health Management Resources (HMR) daily (110 kcal per serving)

Activity: Optional. A game board aiming to increase number of steps. Behaviour: praise problem solving, reward system. Carers could assist if needed.

Maintenance: 6 months of less intensive meetings. Weight loss could continue if wanted.
Follow up

6 months

Results

$(n=73)$ Mean weight change, $k g:-6^{b}$

Mean BMl change $\mathrm{kg} / \mathrm{m}^{2} \approx-2.7 \%$ mean weight loss: 6.3

data are mean values (SD)

range not reported. 


\section{Author details}

${ }^{1}$ College of Medical, Veterinary and Medical and Life Sciences, University of Glasgow, Glasgow G31 2ER, UK. ²Learning Disabilities Psychiatry, College of Medical Veterinary and Life Sciences, Institute of Mental Health \& Wellbeing University of Glasgow, Glasgow G12 0XH, UK. ${ }^{3}$ Human Nutrition, College of Medical, Veterinary and Medical and Life Sciences, University of Glasgow,

Glasgow Royal Infirmary, Level 2, New Lister Building, Glasgow G31 2ER, UK

Received: 10 November 2014 Accepted: 24 November 2014

Published: 22 December 2014

\section{Reference}

1. Spanos D, Melville CA, Hankey CR: Weight management interventions in adults with intellectual disabilities and obesity: a systematic review of the evidence. Nutr J 2013, 12:132.

doi:10.1186/1475-2891-13-123

Cite this article as: Spanos et al.: Correction: weight management interventions in adults with intellectual disabilities and obesity: a systematic review of the evidence. Nutrition Journal 2014 13:123.

\section{Submit your next manuscript to BioMed Central and take full advantage of:}

- Convenient online submission

- Thorough peer review

- No space constraints or color figure charges

- Immediate publication on acceptance

- Inclusion in PubMed, CAS, Scopus and Google Scholar

- Research which is freely available for redistribution 